\title{
Monitored anaesthesia care in elderly patient - a prospective descriptional study
}

\author{
Abdullah Al Maruf ${ }^{1 *}$, Kazi Ashkar Latif ${ }^{1}$, Iqbal Hossain Chowdhury ${ }^{2}$, Md. Mustafa Kamal ${ }^{3}$ \\ *1.Graded Specialist in Anaesthesiology, Department of Anaesthesia and Intensive care, CMH, Dhaka, 2.Associate Professor, \\ Department of Anaesthesia, Analgesia and Intensive care unit, BSMMU Dhaka 3. Assistant Professor, Department of \\ Anaesthesia, Analgesia and Intensive care unit, BSMMU Dhaka
}

* Corresponding author: Dr. Abdullah Al Maruf, E-mail: iqbal-hossain-56@gmail.com

\begin{abstract}
Background: In elderly patients for some diagnostic, therapeutic and surgical procedure, Monitored anaesthesia care (MAC) may be an anaesthetic option for them.

Aim and objective: This prospective study was designed to assess the efficacy, safety, and tolerability of MAC in elderly patients.

Method: This study was performed on elderly patients of both sex, age from 50 years and above, scheduled to undergo different therapeutic, diagnostic and surgical procedures. The patient's characteristics, pre-anaesthetic problems, anaesthetic techniques, anaesthetic agents, anesthetic time, MAC procedure and complications were assessed.

Result: They involved mainly hypertension (26.49\%) and diabetes mellitus (21.78\%). Almost all procedure was done under sedation (70.38\%), local anaesthesia (22.71\%) and under only monitoring without sedation or local anaesthesia (6.90\%). There were no serious adverse events reported in any patients during MAC. The common complications were arrhythmia (9.60\%), vomiting (7.36\%) and desaturation (4.56\%), hypertension (5.64\%). Complications were minor, transient and promptly managed and corrected. MAC duration was ranged from 15 to 180 minutes. The mean procedure time was $35.1 \pm 12.5$ minutes. Majority of cases were completed within 30 minutes (51.21\%).
\end{abstract}

Conclusion: In properly selected elderly patients, MAC is a safe and effective method of providing intraoperative care for some common diagnostic, therapeutic, and operative procedures.

Keywords: Monitored anaesthesia care (MAC), elderly, sedation, monitoring.

(Journal of BSA, 2010; 23(1): 19-24)

\section{Introduction}

Monitored Anaesthesia Care (MAC) is a specific anaesthesia service in which an anaesthesiologist has been requested to participate in the care of a patient undergoing a diagnostic or therapeutic procedure. Indication for monitored anaesthesia care includes the nature of the procedure, the patient's clinical condition and the potential need to convert to a general or regional anaesthetia. ${ }^{1}$ MAC includes all aspects of anaesthesia care; a pre- procedure visit, intra- procedure care and post procedure anaesthesia management. During MAC, the anaesthesiolgist directs a number of specific services, including but not limited to; (a) diagnosis and treatment of clinical problems those occur during the procedure, (b) support of vital functions, (c) administration of sedatives, analgesics, hypnotics, anaesthetic agents or other medications as necessary for patients safety, (d) psychological support and physical comfort, and (e) provision of other medical services as needed to complete the procedure safely. MAC may include varying levels of sedation, analgesia and anxiolysis as necessary. The provider of MAC must be prepared and qualified to convert general anaesthesia when necessary. If the patient loses consciousness and the ability to respond purposefully, the anaesthesia care is a general anaesthetic, irrespective of whether airway instrumentation is required or not. 
As the member of elderly patients undergoing surgery continues to rise, it is important to consider anaesthetic option that minimize physiological stress in patient. MAC, sedation and monitoring are an attractive option for certain common procedures. ${ }^{2}$ However those anaesthesiologists administering MAC must consider the normal functional reserve in elderly patient. ${ }^{3}$ These includes (a) loss of normal compensation for the stress of hypovolaemia, (b) decreased peripheral vascular resistance, (c) altered mental status and, (e) reduced response to hypoxia and hypercarbia associated with periprocedure and sedated state in this population. Because of changes in body composition, as well as renal and hepatic function, the time to onset and offset of even short acting sedatives will be prolonged. ${ }^{4}$ There is also extreme variability in the response to sedative among these patients, cautions must be exercised through full monitoring of intra-operative and peri-operative mental status, oxygenation, and perfusion status. Elderly patients, scheduled for MAC, vigilance is necessary to identify co-morbid states, which increase incidence with age and often present, atypically.

This prospective descriptional study was designed to assess the efficacy, safety, and tolerability of MAC in elderly patients undergoing diagnostic, therapeutic, and surgical purposes under sedation, local anaesthesia or with only monitoring without any sedation or local anaesthesia.

\section{Materials and Methods}

We performed a prospective descriptional study on elderly patients of both sex, age from 50 years and above, scheduled to undergo different therapeutic, diagnostic and surgical procedures at CMH, Dhaka, Gastro Liver Clinic, Dhaka, and IBN SINA Medical Imaging Centre, Dhaka in one calendar year from July 2005 to June 2006. Patients with anatomic airway abnormalities, severe cardiovascular and respiratory disease and severe psychological problem were excluded from the study. During pre-procedural assessment, every patient underwent thorough physical examination with ASA classifications. Total MAC procedure was explained to every patient and informed consent was taken. A baseline pulse, blood pressure, respiratory rate, ECG and $\mathrm{SpO}_{2}$ were recorded.
Anaesthesiologist was constantly available throughout the diagnostic, therapeutic or surgical procedure to provide MAC. Patient's level of consciousness, heart rate, blood pressure, respiratory rate, $\mathrm{SpO}_{2}$ and electrocaridiography were monitored through out the whole procedure. A full set of resuscitation equipments including suction apparatus, oxygen, a bag valve mask, age appropriate airway, resuscitation drugs and defibrillator were available throughout sedation and recovery to combat any adverse event. Any serious adverse events as well as side effects during MAC like desaturation $\left(\mathrm{SpO}_{2}\right.$ less than $\left.93 \%\right)$ hypertension (systolic BP more than $30 \%$ of baseline record), hypotension (systolic BP less than $90 \mathrm{~mm}$ of $\mathrm{Hg}$ ), arrhythmia, vomiting, agitation and nightmares were observed, recorded and managed.

Data from anesthetic, procedure records and history charts of patients were recorded. The general data included age, sex, height, body weight, and ASA physical status. The anesthetic data encompassed pre-anaesthetic problems, different procedures, choice of anesthesia, variety of drug usage, duration of procedure and complications evolved during procedure. Results were expressed as mean \pm standard deviation (SD) or percentage (\%) where appropriate.

\section{Results}

Characteristics of patients were shown in table I. There were 2144 procedures performed during the study period, majority patients were male (53.07\%), mean age was $64.56 \pm 6.78$ years. Age groups between 50 to 70 years were $85.95 \%$, ASA physical status between I and II were $90.8 \%$. Pre-anaesthetic problems were shown in table II. There were 1331 patients in less than ASA-II classification but some had multiple diseases and total 1428(66.60\%) preanaesthetic problems found in 2144 patients. They involved mainly hypertension (26.49\%) and diabetes mellitus (21.78\%). Other problems were heart disease(7.78\%); coronary artery disease, valvular heart disease, liver disease(2.09\%); cirrhosis, respiratory disease(3.21\%); chronic obstructive pulmonary disease, asthma, renal disease (2.65\%); chronic renal failure, haematologic disease $(1.95 \%)$; anaemia, and others $(0.60 \%)$; electrolyte imbalance. Details of different diagnostic and therapeutic Procedures done under MAC were shown in table III. Almost all procedure was done under sedation 
(70.38\%), local anaesthesia (22.71\%) and under only monitoring without sedation or local anaesthesia (6.90\%). The details of sedative agents, narcotic drugs and local anaesthetics were shown in table IV. There were no serious adverse events reported in any patients during MAC. Complications during procedure and recovery were observed, recorded, and shown in table $\mathrm{V}$. The common complications were arrhythmia (9.60\%), vomiting (7.36\%) and desaturation (4.56\%), hypertension (5.64\%). Other complications were hypotension (0.97\%) and

\section{Table I}

Demographic data of different study group

\begin{tabular}{lcc}
\hline Characteristics & Number & Percentage \\
\hline Sex & & \\
$\quad$ Male & 1138 & $53.07 \%$ \\
$\quad$ Female & 1006 & $46.92 \%$ \\
Age(years) & & \\
$\quad 50-59$ & 1007 & $46.96 \%$ \\
$60-69$ & 834 & $38.99 \%$ \\
$70-79$ & 235 & $10.96 \%$ \\
$80-89$ & 57 & $2.65 \%$ \\
$\quad>90$ & 11 & $0.51 \%$ \\
Mean age \pm SD(years) & $64.56 \pm 6.78$ \\
ASA physical status & \multicolumn{2}{|}{} \\
I 813 & $37.91 \%$ & \\
II & 1134 & $52.89 \%$ \\
III & 128 & $5.97 \%$ \\
IV & 69 & $3.21 \%$ \\
\hline
\end{tabular}

There were 2,144 cases, but age and ASA physical status were not the same.

Table II Preanaesthetic problem

\begin{tabular}{lcc}
\hline Preanaesthetic problem & Number & Percentage \\
\hline Hypertension & 568 & $26.49 \%$ \\
Diabetes Mellitus & 467 & $21.78 \%$ \\
Heart disease & 167 & $7.78 \%$ \\
Respiratory disease & 69 & $3.21 \%$ \\
Renal disease & 57 & $2.65 \%$ \\
Hepatic disease & 45 & $2.09 \%$ \\
Haematologic disease & 42 & $1.95 \%$ \\
Others & 13 & $0.60 \%$ \\
\hline Total & 1428 & $66.60 \%$ \\
\hline
\end{tabular}

agitation and nightmares (1.77\%), and local anaestheic toxicity $(0.13 \%)$. Complications were minor, transient and promptly managed and corrected. Details of duration of MAC were shown in table VI. The MAC duration was ranged from 15 to180 minutes. The mean procedure time was $35.1 \pm 12.5$ minutes. Majority of cases were completed within 30 minutes $(51.21 \%)$, and between 30 to 60 minutes $(40.48 \%)$. Other procedures completed between 60 - 89 minutes $(7.78 \%)$ and more then 90 minutes required in $0.51 \%$ cases.

Total number of cases 2144 and there were 1331 patients in less than ASA-II classification but some had multiple diseases and total 1428 preanaesthetic problem found in them.

Table III

Procedures done under MAC

\begin{tabular}{lcc}
\hline Procedure & Number & Percentage \\
\hline Under sedation & 1509 & $70.38 \%$ \\
ERCP & 237 & $11.05 \%$ \\
Upper GI endoscopy & 356 & $16.60 \%$ \\
Lower GI endoscopy & 307 & $14.31 \%$ \\
MRI & 238 & $11.10 \%$ \\
CT scan & 124 & $5.78 \%$ \\
Radiotherapy & 67 & $3.12 \%$ \\
Chemotherapy & 34 & $1.58 \%$ \\
Wound dressing & 146 & $6.80 \%$ \\
Under local anaesthesia & 487 & $22.71 \%$ \\
Cataract surgery & 352 & $16.41 \%$ \\
Excision of cyst/lipoma & 103 & $4.80 \%$ \\
Wound repair/dressing & 32 & $1.49 \%$ \\
Without sedation or local & 148 & $6.90 \%$ \\
anaesthesia & & \\
Upper GI endoscopy & 34 & $1.58 \%$ \\
Lower GI endoscopy & 23 & $1.07 \%$ \\
MRI & 27 & $1.25 \%$ \\
CT scan & 17 & $0.79 \%$ \\
Radiotherapy & 09 & $0.41 \%$ \\
Chemotherapy & 07 & $0.32 \%$ \\
Wound dressi3ng & 31 & $1.44 \%$ \\
\hline
\end{tabular}

Total number of cases 2144 
Table-IV

Anaesthesia related data

\begin{tabular}{lcc}
\hline Data & Number & Percentage \\
\hline Sedative agents & & \\
$\quad$ Diazepam & 245 & $11.42 \%$ \\
Midazolam & 234 & $10.91 \%$ \\
$\quad$ Propofol & 108 & $5.03 \%$ \\
$\quad$ Ketamine & 370 & $17.25 \%$ \\
Narcotics & & \\
Pethidine & 189 & $8.81 \%$ \\
Morphine & 121 & $5.64 \%$ \\
Fentanyl & 145 & $6.76 \%$ \\
Nulbuphine & 97 & $4.52 \%$ \\
Local anaesthetics & 487 & $22.71 \%$ \\
2\%Lignocaine(plain) & 152 & $7.08 \%$ \\
2\%Lignocaine with & 31 & $1.44 \%$ \\
$\quad$ adrenaline(1:200000) & & \\
0.5\%Bupivacaine(plain) & 175 & $8.16 \%$ \\
0.5\%Bupivacaine mixed & 121 & $5.64 \%$ \\
$\quad$ with 2\% lignocaine & & \\
\hline
\end{tabular}

Total number of cases 2144

Table V

$M A C$ related complications

\begin{tabular}{lcc}
\hline Complications & Number & Percentage \\
\hline Desaturation & 97 & $4.56 \%$ \\
Hypertension & 121 & $5.64 \%$ \\
Hypotension & 21 & $0.97 \%$ \\
Arrhythmia & 206 & $9.60 \%$ \\
$\quad$ Tachycardia & 145 & $6.76 \%$ \\
$\quad$ Bradycardia & 47 & $2.19 \%$ \\
$\quad$ Other arrhythmias & 14 & $0.65 \%$ \\
Vomiting & 158 & $7.36 \%$ \\
Agitation and nightmares & 38 & $1.77 \%$ \\
Local anaesthetic toxicity & 3 & $0.13 \%$ \\
\hline Total & 850 & $39.64 \%$ \\
\hline
\end{tabular}

Total number of cases 2144
Table VI

Duration of $M A C$

\begin{tabular}{lcc}
\hline $\begin{array}{l}\text { Duration of time } \\
\text { (minutes) }\end{array}$ & Number & Percentage \\
\hline$<30$ & 1098 & $51.21 \%$ \\
$30-59$ & 868 & $40.48 \%$ \\
$60-89$ & 167 & $7.78 \%$ \\
$>90$ & 11 & $0.51 \%$ \\
Mean procedure & $35.1 \pm 12.5$ & \\
time+SD (minutes) & & \\
Total number of cases 2144 & \\
&
\end{tabular}

The Monitored Anesthesia Care (MAC) phrase refers to instances in which an anesthesiologist has been called upon to provide specific anesthesia services to a particular patient undergoing a planned procedure, in connection with which a patient receives sedation, local anesthesia or in some cases no anesthesia at all. In such a case, the anesthesiologist is providing specific services to the patient and is in control of the patient's nonsurgical or medical care, including the responsibility of monitoring of the patient's vital signs, and is available to administer anesthetics or provide other medical care as appropriate.

Purpose of MAC is to provide the patient with anxiety relief, amnesia, pain relief, comfort and safety during any therapeutic, diagnostic, and surgical procedure. MAC can be requested for patients undergoing uncomfortable procedures and minor surgeries, which do not require general anaesthesia. ${ }^{5}$ At one time, the term local standby described the role of anaesthesiologists in these cases. Monitored Anaesthesia Care has now replaced this term, as the anaesthesiologist should be continually monitoring the patient during surgery or procedure not just standby. ${ }^{6}$ There are few anaesthetic techniques those are used in younger patient cannot be used to anaesthetize the elderly patient. For elderly patients for some diagnostic, therapeutic including some surgical procedures MAC may be a better option for them. ${ }^{2}$ In general, elderly patients are frailer, with greater likelihood of peri-operative complications. However even in the absence of a specific organbased disease process, anaesthesia for the elderly may require an alteration in technique to the effects 
that the aging process, the disease process, and any residual effects of previous illness have hand on the elderly patient and tailor the anaesthetic technique accordingly. ${ }^{7}$ The appearance and activity of the patient are thought to be more relevant than the actual age e.g. 90 years old may present less risk than frail 70 years old. ${ }^{8}$ Cardiopulmonary and other disease that are more frequent in older patients have been regarded as the major risk factors for the complications associated with sedation and procedures. ${ }^{9,10,11}$ In this study we performed MAC for surgeries and different diagnostic and therapeutic procedures with or without sedation or under local anaesthesia in elderly patients. There were several studies on surgeries and diagnostic and therapeutic procedures under MAC. They prove MAC can be successfully achieved during surgeries like cataract surgery, skin surgery and diagnostic procedure like endoscopies and imaging procedures. ${ }^{12,13,14} \mathrm{MAC}$ combines intravenous sedation with local anaesthesia, nerve blocks or with endoscopies. ${ }^{15,16}$ There were no special anaesthetic techniques needed for MAC. Different sedation regimens are available. In this study, we used benzodiazepines, opioids, propofol and ketamine in titrated low doses. The primary disadvantages of sedation are the lack of airway control and the threat of aspiration or airway obstruction. To minimize the risks, the anaesthesiologist must titrate medications carefully to maintain spontaneous respirations while maintaining an anaesthetic depth, allowing the patient to remain comfortable. Careful selection and administration of medications is essential in producing the desired and optimal intra-procedure anaesthetic effects and post procedure outcomes. Sedatives and opioids can also make patient drowsy and may cause desaturation.

Duration of procedure should not take prolong time as related with patient's discomfort and complications. ${ }^{17}$ In this study, most of the procedure completed within 60 minutes duration. Complications may arise during MAC in elderly patients. Causes are co-existing diseases in elderly, sedation, local anaesthetic and the procedure itself. Hypoxia or desaturation are common with sedation with benzodiazepine and opioids. ${ }^{18}$ Arrhythmias are usually observed with coexisting cardiac diseases and with upper GIT endoscopies and cataract surgeries. ${ }^{19,20}$ Patient undergo MAC are never mend to be without recall. ${ }^{21}$ Whether or not a patient remembers the procedure depends on the type of medications uses, dosage, patients physiology, and other factors. Many patients undergoing MAC do not remember the experiences. In this study, no serious complications were observed. Incidence of complications associated with sedation, procedure and local anaesthetics found less and easily manageable with available resources.

\section{Conclusion:}

Concluded in properly selected elderly patients, MAC is a safe and effective method of providing intraoperative care for some common diagnostic, therapeutic, and surgical procedures. However, few complications may arise during procedure but they were minor, transient and promptly managed and corrected.

\section{References:}

1. ASA position on Monitored Anesthesia Care. Approved by the House Delegates September 2008.

2. Margaret Ekstein, Doron Gavish, Tiberia Ezri, Avi Weinbroum. Monitored Anesthesia Care in the elderly: Guidelines and Recommendations. Drugs Aging 2008; 25(6):477-500.

3. Raymond R. Anesthetic management of the elderly patient. 53rd ASA Annual Meeting Refresher Course Lectures no. 2002; 321: 1-7.

4. Muravichik S. Pharmacological changes of aging. $53^{\text {rd }}$ ASA Annual Meeting Course Lectures 2002; 1-7.

5. Rego M, White PF. Monitored Anesthesia Care. RD Miller(ed). Miller's Anesthesia $5^{\text {th }}$ ed. Philadelphia. Churchill Livingstone, 2000:1452-1467.

6. G. Edward Morgan, Jr. Maged S. Mikhali, Michael J. Murray. Clinical Anesthesiology. 4th ed. USA. McGraw-Hill, 2006:831.

7. David Murray, Chris Dodds. Perioperative care of the elderly. Anaesthesia, Critical Care \& Pain 2004;4(6):193-196.

8. Steven MY, Nicholas PH, Gary BS. Anaesthesia and Intensive care A-Z. 3rd ed. UK Butterworth Heinemann, 2004:175-176. 
9. Nagengast EM. Sedation and monitoring in gastrointestinal endoscopy. Scand J Gastroenterol 1993;200:28-32.

10. Bell GD, McCloy RF, Campell D, et el. Recommendations for standards of sedation and patient monitoring in gastrointestinal endoscopy. Gut 1991; 32:823-827.

11. Bell GD. Premedication and intravenous sedation for upper gastrointestinal endoscopy. Aliment Pharmacol Ther 1990; 4: 103-122.

12. Bing J, McAuliffe MS, Lupton JR. Regional anesthesia with monitored anesthesia care for dermatologic laser surgery. Dermatol Clin 2002;20(1):123-124.

13. Rosenfeld SI, Litinsky SM, Snyder DA, Plosker H, Astrove AW, Schiffman J. Effectiveness of Monitored Anesthesia Care in Cataract surgery. Ophthalmology 1999; 106:1256-1261.

14. Malhotra S, Dutta A, Gupta A. Monitored anaesthesia in elderly ophthalmic patients. The Lancet 2003; 359: 532.
15. Badrinath S, et el. The use of ketamine - propfol combination during Monitored Anesthesia Care. Anesth. Analg 2000; 90:858-862.

16. Michael Mercandetti. Anethesia, local with sedation. Medicine. Update on March 7, 2008.

17. Warner D, Warner M. Anesthetic risk and the elderly. Syllabus on Geriatric Anesthesiology. ASA. 2002:1-4.

18. Anh - thuy Nguyen. Monitored Anesthesia Care. The Internet Journal of Health 2000; 1

19. Lee JF, Leung JWC Cotton PB. Acute cardiovascular complications of endoscopy: prevalence and clinical characteristics. Dig Dis 1995; 13(2): 130-135.

20. Lieberman DA, Wuerker CK, Katon RM. Cardiopulmonary risk of endoscopic diameter and systematic sedation. Gastroenterology 1985; 88: 468-472.

21. Lekpraser Varinee. "Preanaesthetic Assessment of Patient Who Reports Previous Introperative Awareness." Anaesthesiology News 2008:35-38. 